Editorial

\title{
Is copeptin ready for clinical use in a dual-marker strategy with high-sensitivity cardiac troponin to rule-out acute myocardial infarction?
}

\author{
Ana Teresa Timóteo $*, 1$ \\ NOVA Medical School, Lisbon, Portugal \\ Santa Marta Hospital, Centro Hospitalar Universitário Lisboa Central, Portugal
}

\section{A R T I C L E I N F O}

\section{Article history:}

Received 5 November 2018

Accepted 21 November 2018

Available online 22 November 2018

Several new biomarkers have been described to improve diagnostic accuracy of many diseases. Copeptin is produced in the neurohypophysis and is a stable peptide derived from the C-terminal part of the arginine vasopressin prohormone [1]. Vasopressin has a very short halflife and it is very difficult to measure being copeptin an alternative quantitative biomarker, because it is released in a proportional manner with biologically active vasopressin [1]. Copeptin plasma concentrations increase in response to endogenous stress in several clinical situations including acute cardiovascular conditions, such as acute myocardial infarction (AMI), myocarditis and heart failure [2-4]. However, it lacks specificity because it can also increase in other non-cardiac acute situations. It is used as a non-specific marker of acute and severe disease. As a single variable, it has only modest accuracy for AMI.

A rapid rule-in and rule-out strategy in the emergency department (ED) is highly desirable, particularly in non-ST elevation AMI. Not only because it can speed the treatment but also because patients without AMI can be discharged earlier, reducing important constrains in the ED, such as crowding and substantial use of resources (prolonged ECG monitoring and serial blood draws). Recently, several algorithms have been validated and are currently recommended in European guidelines [5]. High sensitivity cardiac troponins (hs-cTn) are the recommended biomarkers and patients can be safely ruled-out within 1 to $3 \mathrm{~h}$ after hospital admission. However, conventional and less sensitive cTn assays

DOI of original article: https://doi.org/10.1016/j.ijcard.2018.10.084.

* Hospital Santa Marta, Centro Hospitalar Universitário Lisboa Central, Rua Santa Marta, Lisbon, Portugal.

E-mail address: ana_timoteo@yahoo.com.

This author takes responsibility for all aspects of the reliability and freedom from bias of the data presented and their discussed interpretation. are still widely used in several countries, particularly in institutions outside of Europe. Conventional cTn algorithms, require longer waiting times and serial measurements up to $6-12 \mathrm{~h}$ after presentation.

Copeptin has been suggested as a potential biomarker to evaluate patients with acute chest pain and to safely rule-out patients in the ED. While cTn is released into the circulation in a time-dependent fashion, elevations of copeptin occur very early in AMI [2,3]. Since the initial observations by Reichlin that copeptin is associated with AMI, several studies tested the feasibility and safety of a dual-marker strategy (DMS), currently supported by several robust studies [6,7]. However, the sensitivity of the cTn assay can determine the incremental clinical value of copeptin [2,7-9]. In fact, the incremental benefit has only been confirmed with conventional cTn, and it was minor with more sensitive assays. Also, the area under curve of copeptin in combination with hc-cTn at presentation is lower when compared to hs-cTn alone at presentation and $1 \mathrm{~h}$ after ED presentation. These results showed that copeptin was only effective when less sensitive conventional cTn were used. Due to the lack of evidence to demonstrate that there was a significant benefit with the DMS with hs-cTn, the use of copeptin was only recommended with lower sensitivity troponins. A DMS of cTn level below the 99th percentile and a copeptin level below $10 \mathrm{pmol} / \mathrm{L}$ has a very high negative predictive value, particularly when used in conjunction with full clinical assessment, including a 12-lead ECG [6-8]. Studies that tested hs-cTn with a cut-off value below the 99th percentile showed the same negative predictive value as the combination of hs-cTn at the 99th percentile together with a negative copeptin $[8,9]$. When using the 99th percentile as cut-off value for hs-cTn, it is required further studies to convincingly established a clinically relevant increase in diagnostic performance with a DMS to justify routine clinical use. The cut-off value for copeptin is also a matter of debate. Whit the first-generation assays, the recommended cut-off was $14 \mathrm{pmol} / \mathrm{L}$. With more recent and higher sensitivity assays, a cutoff of $10 \mathrm{pmol} / \mathrm{L}$ is recommended [2].

In the present issue of International Journal of Cardiology, MuellerHennessen et al presented a manuscript that compares a combined DMS with copeptin and hs-cTnT (99th percentile) at presentation with other rapid rule-out algorithms in 922 patients presenting to the ED with suspected AMI [10]. The authors tested several cut-offs for both copeptin and hs-cTnT. They showed lower rule-out rates with the DMS and higher negative predictive values and sensitivities, 
particularly in comparison with the hs-cTnT 99th percentile. However, addition of clinical risk criteria allowed an increase in sensitivity up to $100 \%$ but also with significant reductions in rule-out rates. All strategies were safe, with very low event rates $(0.2-0.3 \%)$ at 30 -day follow-up. The authors concluded that a DMS might be an alternative, but it depends on the applied copeptin cut-off (with higher rule-out rates with the cut-off of $20 \mathrm{pmol} / \mathrm{L}$, with similar negative predictive values) and addition of clinical low-risk criteria.

There are however presently, several important barriers for the implementation of this strategy. Currently, this requires two different diagnostic platforms to measure both hs-cTnT and copeptin. This is an important inconvenient because it requires an additional analyzer. Also, the time span between blood draw and results availability might take longer than the time required for other rule-out algorithms. In theory, and if these problems are solved in a near future, this DMS is possibly associated with cost savings. However, the exact costeffectiveness is unknown. Adequately designed health economic studies are needed to address this subject.

Another important point to mention is that this study confirmed the importance of adequate clinical judgment for a correct rule-out. The inclusion of clinical criteria increased the negative predictive value and sensitivity. For that reason, it is important that independently of the strategy chosen, all clinical information must be appropriately assessed by ED physicians (including detailed assessment of chest pain and the ECG) to avoid inappropriate rule-out and discharge of AMI patients. Another important point is that copeptin does not increase in the presence of unstable angina [2]. Therefore, if we ruleout AMI, it does not mean that the patients do not have significant coronary artery disease, and if symptoms are typical, further testing might be warranted, usually in an ambulatory setting.

With the present data, we can conclude that a DMS with copeptin and hs-cTnT might be useful, but it is not ready yet to be implemented in routine clinical practice. It requires comprehensive health economic analysis and practical solutions regarding laboratory testing.

\section{Conflict of interests}

The author reports no relationships that could be construed as a conflict of interest.

\section{References}

[1] Christ-Crain, W. Fenske, Copeptin in the diagnosis of vasopressin-dependent disorders of fluid homeostasis, Nat. Rev. Endocrinol. 12 (2016) 168-176.

[2] C. Mueller, M. Mockel, E. Giannitsis, et al., Use of copeptin for rapid rule-out of acute myocardial infarction, Eur. Heart J. Acute Cardiovasc. Care 7 (2018) 570-576.

[3] S.Q. Khan, O.S. Dhillon, R.J. O'Brien, et al., C-terminal provasopressin (copeptin) as a novel and prognostic biomarker in acute myocardial infarction: Leicester Acute Myocardial Infarction Peptide (LAMP) study, Circulation 115 (2007) 2103-2110.

[4] J. Silva Marques, H. Luz-Rodrigues, C. David, et al., Biomarkers of functional class in systolic heart failure: the relevance of copeptin, Rev. Port. Cardiol. 31 (2012) 701-710.

[5] M. Roffi, C. Patrono, J.P. Collet, et al., 2015 ESC Guidelines for the management of acute coronary syndromes in patients presenting without persistent ST-segment elevation: Task Force for the Management of Acute Coronary Syndromes in Patients Presenting Without Persistent ST-Segment Elevation of the European Society of Cardiology (ESC), Eur. Heart J. 37 (2016) 267-315.

[6] T. Reichlin, W. Hochholzer, C. Stelzig, et al., Incremental value of copeptin for rapid rule-out of acute myocardial infarction, J. Am. Coll. Cardiol. 54 (2009) 60-68.

[7] T. Raskovalova, R. Twerenbold, P.O. Collinson, et al., Diagnostic accuracy of combined cardiac troponin and copeptin assessment for early rule-out of myocardial infarction: a systematic review and meta-analysis, Eur. Heart J. Acute Cardiovasc. Care 3 (2014) 18-27.

[8] K. Wildi, C. Zellweger, R. Twerenbold, et al., Incremental value of copeptin to highly sensitive cardiac troponin I for rapid rule-out of myocardial infarction, Int. J. Cardiol. 190 (2015) 170-176.

[9] C. Mueller, E. Giannitsis, M. Mockel, et al., Rapid rule-out of acute myocardial infarction: novel biomarker-based strategies, Eur. Heart J. Acute Cardiovasc. Care 6 (2017) 218-222.

[10] M. Mueller-Hennessen, B. Lindahl, E. Giannitsis, et al., Combined testing of copeptin and high-sensitivity cardiac troponin $\mathrm{T}$ at presentation in comparison to other algorithms for rapid rule-out of acute myocardial infarction, Int. J. Cardiol. 276 (2019) 261-267. 